\section{(6) \\ OPEN ACCESS}

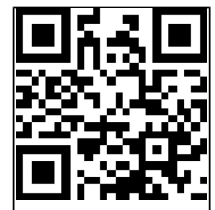

Open Access

can to access more

free content

- Additional material is published online only. To view please visit the journal online (http://dx.doi.org/10.1136/ jnnp-2012-304332).

${ }^{1}$ Department of Clinical Neuroscience, Center for Molecular Medicine, Karolinska Institutet, Stockholm, Sweden ${ }^{2}$ Department of Neurology, Medical Faculty, HeinrichHeine-University, Düsseldorf,

Germany

${ }^{3}$ Translational Medicine, Biogen Idec, Inc., Cambridge, Massachusetts, USA

${ }^{4}$ Clinical Virology, Department of Infectious Diseases, Institute of Biomedicine, University of Gothenburg, Gothenburg, Sweden

${ }^{5}$ Elan Pharmaceuticals, San Francisco, California, USA ${ }^{6}$ Department of Medicine, Center for Molecular Medicine, Karolinska Institute, Stockholm, Sweden

\section{Correspondence to} Dr Clemens Warnke, The Multiple Sclerosis Research Group, Department of Clinical Neuroscience, Center for Molecular Medicine, Karolinska Institutet, Stockholm SE-171 76, Sweden; clemenswarnke@gmail.com

Received 9 October 2012 Revised 4 February 2013 Accepted 5 February 2013 Published Online First 5 March 2013

\section{SLinked}

- http://dx.doi.org/10.1136/ jnnp-2012-304777

\footnotetext{
To cite: Warnke $C$, Ramanujam R, Plavina T, et al. I Neurol Neurosurg Psychiatry 2013;84: 1199-1205.
}

\title{
Changes to anti-JCV antibody levels in a Swedish national MS cohort
}

\author{
Clemens Warnke, ${ }^{1,2}$ Ryan Ramanujam, ${ }^{1}$ Tatiana Plavina, ${ }^{3}$ Tomas Bergström, ${ }_{1}^{4}$ \\ Susan Goelz, ${ }^{5}$ Meena Subramanyam, ${ }^{3}$ Ingrid Kockum, ${ }_{1}^{1}$ Afsar Rahbar, ${ }^{6}$ \\ Bernd C Kieseier, ${ }^{2}$ Carolina Holmén, ${ }^{1}$ Tomas Olsson, ${ }^{1}$ Jan Hillert, ${ }^{1}$ \\ Anna Fogdell-Hahn ${ }^{1}$
}

\section{ABSTRACT}

Background The anti-JC virus (JCV) antibody status has been introduced to stratify patients with multiple sclerosis (MS) for higher or lower risk of progressive multifocal leukoencephalopathy (PML).

Objective To assess the potential utility of anti-JCV antibody levels for earlier diagnosis or prediction of PML. Methods An analytically validated antibody assay was used to determine serological status, normalised optical density values, and dilution titres for anti-JCV antibodies. The method was applied to stored sera of 1157 patients with MS including five cases of PML, all enrolled in the Swedish pharmacovigilance study for natalizumab (NAT). Anticytomegalovirus (CMV) and antivaricella-zoster (VZV) antibody levels served as controls.

Results Prior to treatment with NAT, anti-JCV antibody levels were stable in the anti-JCV positive patients. During therapy, a slight decrease in anti-JCV and antiVZV antibody levels, but not anti-CMV antibody levels, was observed. All five patients who developed PML showed a mild to moderate increase in anti-JCV antibody levels at time of PML diagnosis; pre-PML samples suggested that this increase might start already prior to diagnosis of PML.

Conclusions Treatment initiation with NAT may lead to a slight decrease in anti-JCV and anti-VZV antibody levels, suggestive of a mild suppressive effect of NAT on antibody levels. Our findings in five cases of PML demonstrate that the onset of PML can be accompanied by increasing anti-JCV antibodies in serum. Monitoring of anti-JCV antibody levels could potentially be used as a tool for prediction or earlier diagnosis of PML during NAT treatment for MS. Further studies are warranted.

\section{INTRODUCTION}

Multiple sclerosis (MS) is a chronic immune-mediated disease of the central nervous system (CNS). The transmigration of activated $\mathrm{T}$ cells from the peripheral blood into the CNS is considered as a crucial step to initiate and maintain this highly debilitating disease, a process in which the $\alpha_{4} \beta_{1}$-integrin (VLA-4) is critically involved. ${ }^{1}$ Natalizumab (NAT) is a humanised monoclonal $\mathrm{IgG}_{4}$ antibody designed to specifically target this step in MS pathogenesis by blocking the $\alpha_{4}$-subunit of VLA-4 on activated T cells. ${ }^{2}$ Although clinically highly efficacious, the occurrence of cases of NAT-associated progressive multifocal leukoencephalopathy (PML), a CNS infection caused by the JC virus $(\mathrm{JCV})$, limits its use. ${ }^{3-5}$ While many aspects of the pathogenesis of PML during NAT treatment still remain obscure, two elements have been recently elucidated: (a) sequencing analyses of the viral genome indicate that patients who develop PML have a pathogenic form of the virus, with specific changes in the regulatory region and the major viral capsid protein VP1 that may facilitate the spread of the virus from the periphery to the brain, ${ }^{6-8}$ (b) the inhibition of immune cell trafficking into the CNS by NAT could allow pathogenic forms of JCV to become established due to the reduced immune surveillance in the CNS. ${ }^{9}$ Consistent with these concepts, infection with JCV is considered a prerequisite for the development of PML. This assumption is supported by the observation that NAT-associated cases of PML tested positive for anti-JCV-IgG antibodies (anti-JCV antibodies) in samples taken prior to (range 6-187 months, $n=64$ ), or around the time of PML diagnosis $(n=101) .{ }^{10-12}$ These findings have resulted in a two-step anti-JCV antibody assay being introduced to stratify patients with MS treated with NAT for lower or higher risk of PML. ${ }^{13-15}$

In Sweden, the patients with MS treated with NAT are included in a postmarketing surveillance study. ${ }^{16}{ }^{17}$ A unique set of prospectively collected blood samples from this nationwide cohort allowed us to assess the potential utility of anti-JCV antibody levels in serum for earlier diagnosis or prediction of PML. For this purpose, anti-JCV antibody status and levels before and after treatment initiation with NAT $(\mathrm{n}=1157)$, and before and at diagnosis of PML $(\mathrm{n}=5)$ were studied. Human anticytomegalovirus (CMV) and antivaricella-zoster-virus (VZV) antibodies served as controls.

\section{PATIENTS AND METHODS}

Patients and samples

The study was performed within the Swedish postmarketing surveillance study for NAT. ${ }^{16}{ }^{17}$ All patients gave written informed consent for this NAT-specific study, approved by the Karolinska Institutet regional ethical committee (KIDnr 2006/1:7, 2006-08-23). Until use, all samples were stored at $-80^{\circ} \mathrm{C}$. The initial cohort tested for anti-JCV antibodies consisted of 1157 MS patients treated with NAT before March 2010. Only patients with a NAT treatment-naïve sample and a second sample (last sample available was selected) during NAT treatment were included in this analysis. Patients with insufficient quantification of anti-JCV antibodies in one of the two samples studied 
(insufficient analysing quality, titres not done), or those previously treated with intravenous immunoglobulin were excluded from the study (total patients excluded: $\mathrm{n}=313$ ); 844 patients remained including five patients with PML. For these patients (NAT-group, median time between sampling 12 months), a time point ( $t 3$ ) just before the first infusion with NAT was compared with a second measurement ( $\mathrm{t} 4$ ) during NAT therapy. As illustrated in online supplementary efigure S1, antibody levels were also studied prior to NAT therapy during treatment with interferon beta (IFN) in a proportion of the same patients. Two subgroups were defined: for the largest, the IFN1-subgroup, samples at $\mathrm{t} 1$ and $\mathrm{t} 3$ were available, whereas the IFN2-subgroup had samples at an additional time point $\mathrm{t} 2$.

\section{Anti-JCV antibody detection and quantification}

A two-step assay to determine anti-JCV antibodies was applied to the samples as published before, ${ }^{10}$ performed at Focus Diagnostics (Cypress, CA, USA) and sponsored by Biogen Idec (Cambridge, Massachusetts, USA). Briefly, in the first step, the optical density (OD) was measured by ELISA, and samples with normalised OD (nOD) values above 0.25 were considered as positive, and below 0.1 as negative. For patients with nOD values between 0.1 and 0.25 (indeterminate zone), the percentage of inhibition was determined in a second step after preincubation with soluble JCV-like particle antigen. Samples with a percentage of inhibition $>40 \%$ and a nOD range between 0.1 and 0.25 were deemed positive (indeterminate-positive), while samples with a percentage of inhibition $<40 \%$ were classified as negative (indeterminate-negative). Sera testing positive for anti-JCV antibodies were diluted in 1:3 dilution steps to determine the anti-JCV antibody titre level.

\section{Anti-CMV and anti-VZV antibody detection}

and quantification

For the anti-CMV antibody status assessment, an ELISA method targeting antigens prepared from the nuclei of CMV-infected fibroblasts (nuclear antigen) was employed as published. ${ }^{18}$ OD values below 0.2 were considered negative. A standard curve was run on each plate for quality control, and paired samples were studied on the same plate.

A recently published ELISA to determine OD values for highly specific IgG-antibodies against a recombinant VZV glycoprotein $\mathrm{E}$ antigen produced in mammalian cells was used to study for anti-VZV antibodies, and paired samples were analysed on the same plate. ${ }^{19} 20$

\section{Statistical analysis}

Calculations were performed in GraphPad Prism V.5.0 for Windows (GraphPad Software, San Diego, California, USA). For categorical values, the $\chi^{2}$ test or the Fisher`s exact test was applied depending on sample size. Continuous unpaired samples were compared applying the Mann-Whitney test or the Kruskal-Wallis test with Dunn's Multiple Comparison test where indicated; paired samples were studied applying the Wilcoxon matched-pairs test. To assess differences in the seropositivity rates across equally spaced age groups, the $\chi^{2}$ test for trend was used. $p$ Values were based on two-tailed statistical tests and considered significant if $<0.05$.

\section{RESULTS}

Seropositivity for anti-JCV antibodies before and during therapy with NAT

As shown in table 1, age and gender were similar for patients in the NAT-group, the IFN-subgroups, and the PML patients. While the NAT-group and the IFN2-subgroup had a similar time between sampling, the latter was longer for the IFN1-subgroup and the PML patients compared with the NAT-group (table 1).

The seropositivity rates for anti-JCV antibodies in the 839 patients of the NAT-group were within the previously reported range, and did not change after treatment initiation with NAT

Table 1 Patient characteristics

\begin{tabular}{|c|c|c|c|c|}
\hline & NAT-group (t3-t4) & IFN1-subgroup (t1-t3)* & IFN2-subgroup (t1-t2)* & PML \\
\hline Total $n$ & 839 & 414 & 170 & 5 \\
\hline Female $n(\%)$ & $587(70)$ & $280(68)$ & $106(62)$ & $3(60)$ \\
\hline Median age (range) & $36(12-63)$ & $37(16-63)$ & $37(16-58)$ & $37(27-44)$ \\
\hline Median $\Delta \mathrm{t}$ months (range) $\dagger$ & $12(1-38)$ & $21(1-70)$ & $13(1-59)$ & $25(18-49)$ \\
\hline Persistent positives $\mathbf{n}(\%$ of all) & $426(51)$ & $202(49)$ & $83(49)$ & \\
\hline Female $\mathrm{n}(\%)$ & $278(65)$ & $123(61)$ & $47(57)$ & \\
\hline Median age (range) & $38(13-63)$ & $39(16-63)$ & $38(16-58)$ & \\
\hline Median $\Delta \mathrm{t}$ months (range) $\dagger$ & $12(1-38)$ & $21(1-70)$ & $12(1-40)$ & \\
\hline Persistent negatives $\mathbf{n}(\%$ of all) & $330(39)$ & $172(42)$ & $75(44)$ & \\
\hline Female $\mathrm{n}(\%)$ & $245(74)$ & $127(74)$ & $53(71)$ & \\
\hline Median age (range) & $35(12-58)$ & $35(17-58)$ & $35(20-54)$ & \\
\hline Median $\Delta \mathrm{t}$ months (range) $\dagger$ & $12(1-38)$ & $19(1-69)$ & $13(2-59)$ & \\
\hline Seroreverters $\mathrm{n}$ ( $\%$ of all) & $45(5)$ & $8(2)$ & $8(5)$ & \\
\hline Female $\mathrm{n}(\%)$ & $36(80)$ & $4(50)$ & $4(50)$ & \\
\hline Median age (range) & $34(14-60)$ & $31(22-45)$ & $39(28-45)$ & \\
\hline Median $\Delta \mathrm{t}$ months (range) $\dagger$ & $23(1-31)$ & $22(2-46)$ & $20(3-43)$ & \\
\hline Sero-converters $\mathrm{n}$ (\% of all) & $38(5)$ & $32(8)$ & $4(2)$ & \\
\hline Female $\mathrm{n}(\%)$ & $28(74)$ & $26(81)$ & $2(50)$ & \\
\hline Median age (range) & $35(20-53)$ & $35(18-53)$ & $33(25-39)$ & \\
\hline Median $\Delta \mathrm{t}$ months (range) $\dagger$ & $24(2-37)$ & $25(1-52)$ & $11(1-39)$ & \\
\hline
\end{tabular}

*IFN1- and IFN2-subgroups are subgroups of the NAT-group defined by samples available prior to NAT therapy at t1 and t2. For details see online supplementary efigure S1. $\dagger \Delta t$ months=time between first and last sample.

NAT, Natalizumab; PML, progressive multifocal leukoencephalopathy. 
(before NAT (t3): 56\%, during NAT (t4): 55\%, p=0.731). The seropositivity increased with age $(\mathrm{p}<0.0001)$, and was higher for men compared with women ( $\mathrm{t} 3$ : women $53 \%$; men: $62 \%$; $\mathrm{p}=0.018$; $\mathrm{t} 4$ : women $52 \%$; men: $63 \% ; \mathrm{p}=0.005)$.

Interestingly, 5.4\% (45/839) of all patients of the NAT-group (table 1), which was $9.6 \%(45 / 471)$ of the positive patients at $\mathrm{t} 3$, reverted to negativity at $\mathrm{t} 4$ during the median 12 months of therapy with NAT. Compared with the 426 persistent positives (positive at $\mathrm{t} 3$ and $\mathrm{t} 4$ ), these seroreverters had lower median nOD values prior to NAT treatment at t3 (0.158 vs 0.479 ; $\mathrm{p}<0.0001$, figure 1A), and most had been classified as indeterminate-positive at the first time point $(91.1 \%(41 / 45)$ vs $23.2 \%(99 / 426) ; \mathrm{p}<0.0001)$.

A similar proportion of the negatives at $\mathrm{t} 3$ demonstrated seroconversion to a positive status at $t 4$ (10.3\% (38/368) of the negative at $\mathrm{t} 3$, or $4.5 \%(38 / 839)$ of all patients). Compared with the 330 persistent negatives (negative at $\mathrm{t} 3$ and $\mathrm{t} 4$ ), the seroconverters had slightly higher $\mathrm{nOD}$ values at $\mathrm{t} 3(0.085$ vs 0.098 ; $\mathrm{p}=0.011$, figure $1 \mathrm{~B}$ ), and there was a trend for a higher proportion of patients classified as indeterminate-negative $(42.1 \%$ $(16 / 38)$ vs $26.7 \%(88 / 330) ; \mathrm{p}=0.057)$. Interestingly, a comparably high proportion $(47.3 \%(18 / 38))$ of the seroconverters during NAT therapy increased by nOD $>0.2$, suggestive of biological, rather than technical change. By contrast, a significantly lower proportion $(6.1 \%(2 / 33) ; \mathrm{p}=0.003)$ of the seroconverters during the IFN therapy (negative at $\mathrm{t} 1$, positive at $\mathrm{t} 2$ and/or $\mathrm{t} 3$ ) increased by $\mathrm{nOD}>0.2$ (figure $1 \mathrm{C}$ ), explaining the lower median change in nOD observed for these patients (figure 1D).

\section{Anti-JCV antibody levels before and during therapy with NAT}

In anti-JCV antibody positive patients, anti-JCV antibody levels as measured in nOD slightly declined in paired samples after the treatment initiation with NAT (Nat-group: median $\triangle \mathrm{nOD}$ : $-0.027 ; \mathrm{p}<0.0001)$, while they were unchanged during the preceding treatment with IFN (IFN1-subgroup: median $\triangle \mathrm{nOD}$ : $+0.015 ; \mathrm{p}=0.105$; IFN2-subgroup: median $\Delta \mathrm{nOD}:-0.001$; $\mathrm{p}=0.979$ ), or showed an upwards trend in the PML patients for the values at diagnosis compared with the pretreatment samples (median $\Delta \mathrm{nOD}+0.284 ; \mathrm{p}=0.062$; figure $2 \mathrm{~A}$ ). Although the changes to the nOD values observed were mild to moderate, all five PML patients showed an increase above the 95 percentile $(\triangle \mathrm{nOD}+0.151)$ of the anti-JCV positive patients of the NAT-group who did not develop PML (figure 2A,B). The proportion of patients who increased in anti-JCV antibodies above this threshold was higher during the therapy with IFN compared with during therapy with NAT (IFN1-subgroup: 15.2\%; IFN2-subgroup: $11.0 \%$ ), but still significantly lower compared with the patients who developed PML (figure 2B).

The median absolute nOD values were similar for the PML patients and the anti-JCV positive patients of the NAT-group prior to NAT-therapy $(0.486$ vs $0.412 ; \mathrm{p}=0.522)$, but higher at diagnosis of PML compared with the values of the NAT-group during NAT ( 0.928 vs $0.371 ; \mathrm{p}=0.008$; figure $2 \mathrm{C}$ ). Only one of the PML patients was outside the 95 percentile of the nOD values of the anti-JCV positive patients of the NAT-group, before and after treatment initiation with NAT.

The 1:3 dilutions titres, as a second measure to quantify anti-JCV antibody levels, confirmed our findings for nOD-values, but proved to be less sensitive for changes in general. Approximately $60 \%$ of the patients remained on the same titre level when comparing two different time points (online supplementary efigure S2A). Nevertheless, the proportion of patients with increasing titres was significantly lower in the NAT-group compared with the PML patients (NAT-group: 5.5\% vs PML patients: $60 \% ; \mathrm{p}=0.002$; online supplementary efigure S2B), and in the NAT group compared with the IFN subgroups (NAT-group: $5.5 \%$ vs IFN1-subgroup: $19 \%$ or IFN2-subgroup: $16 \% ; \mathrm{p}<0.0001$ or $\mathrm{p}=0.001$, respectively). The relatively high proportion of patients who increased in anti-JCV antibody titres during IFN therapy, and the low statistical power due to the low number of PML patients included, explain why only the IFN2-subgroup differed from the PML patients, while the IFN1-subgroup showed the same trend, but did not reach statistical significance (see online supplementary efigure S2B). The median absolute titre values were similar for the PML patients and the anti-JCV positive patients of the NAT-group prior to NAT therapy $(p=0.218)$, but higher in the PML patients at diagnosis $(p=0.013)$. Only one of the PML patients was outside the 95 percentile of the distribution of the absolute titres, before and after treatment initiation with NAT (online supplementary efigure S2C).

The nOD values for anti-JCV antibodies from baseline to diagnosis of PML showed a consistent upwards trend in all the five cases. Compared with the lowest level measured, the trend to increase in nOD started several months prior to diagnosis of PML (figure 3). By contrast, OD values for anti-VZV or anti-CMV antibodies did not exhibit any particular trend, supporting the virus-specificity of the increase in anti-JCV antibodies (figure 3 ).

\section{Anti-VZV and anti-CMV antibody levels prior to and during therapy with NAT}

The antibody response to VZV, as measured in OD values, declined both during the therapy with NAT and IFN, but the amount of the decrease was significantly higher during the NAT-therapy (NAT-group: median $\Delta \mathrm{OD}-0.098, \mathrm{p}<0.0001$; IFN2-subgroup median $\Delta \mathrm{OD}:-0.023, \mathrm{p}=0.003$; figure $4 \mathrm{~A}$ ).

The OD values for anti-CMV antibodies slightly increased during the NAT- or the IFN therapy (NAT-group: median $\triangle \mathrm{OD}$ $+0.061, \mathrm{p}<0.0001$; IFN2-subgroup: median $\Delta \mathrm{OD}$ : +0.061 , $\mathrm{p}=0.128$; figure $4 \mathrm{~B}$ ).

None of the PML patients increased in anti-VZV or anti-CMV antibodies at time of diagnosis, as defined by an increase above the 95 percentile of the OD-changes of the NAT-group.

\section{DISCUSSION}

In this article, we present the results from a large-scale national study $^{16}{ }^{17}$ reporting changes to anti-JCV antibody status and levels in 844 patients with MS, before and during treatment with NAT, including five cases of PML.

Overall, the $55 \%$ seropositivity rate was within the previously published range, and did not change after treatment initiation with NAT. However, a comparably high proportion (around $10 \%$ ) of the negative patients converted to positivity after treatment initiation with NAT, or vice versa. The reversion from positivity to negativity is explained by smaller changes in nOD within the variability of the ELISA assay, and thus, fluctuations around the cut-off account for the majority of these patients. By contrast, among the patients who converted from a negative to a positive status, a proportion of patients $(4.9 \%(18 / 368)$ of the anti-JCV negative patients) demonstrated a relatively strong increase in nOD values, suggestive of a seroconversion which reflects a true change in viral status, rather than merely fluctuations around assay cut-points. Interestingly, the proportion of these patients was significantly lower during the IFN therapy (figure 1). As patients with biological change in serostatus might 
Figure 1 Changes to anti-JCV antibody status after treatment initiation with natalizumab. (A) Seroreverters (positive at $\mathrm{t} 3$ [prior to NAT], negative at 44 [after treatment initiation with NAT]) had lower nOD values at $\mathrm{t} 3$ compared with persistent positives (positive at $\mathrm{t} 3$ and $\mathrm{t} 4$ ). (B) Seroconverters (negative at $\mathrm{t} 3$, positive at 4 ) had slightly higher nOD values at $\mathrm{t} 3$ compared with persistent negatives (negative at $\mathrm{t} 3$ and $\mathrm{t} 4$ ). (C) nOD values shown for all patients negative at $t 1$ [first time point during IFN therapy] who converted to positivity at any time point during the study (t2 [second timepoint during IFN], t3, or t4). (D) Median $\triangle \mathrm{nOD}$ was higher in sero-converters during NAT therapy compared with those converting at $\mathrm{t} 2$ or $\mathrm{t} 3$ during IFN treatment. box: interquartile range; horizontal line: median; whiskers: 5-95 percentile. ${ }^{*} p<0.05 ;{ }^{* *} p<0.01$; $* * * p<0.001$ (Mann-Whitney or Wilcoxon matched pair test); horizontal interrupted line: upper cut-off point of the ELISA at $\mathrm{nOD}=0.25$.
A



B
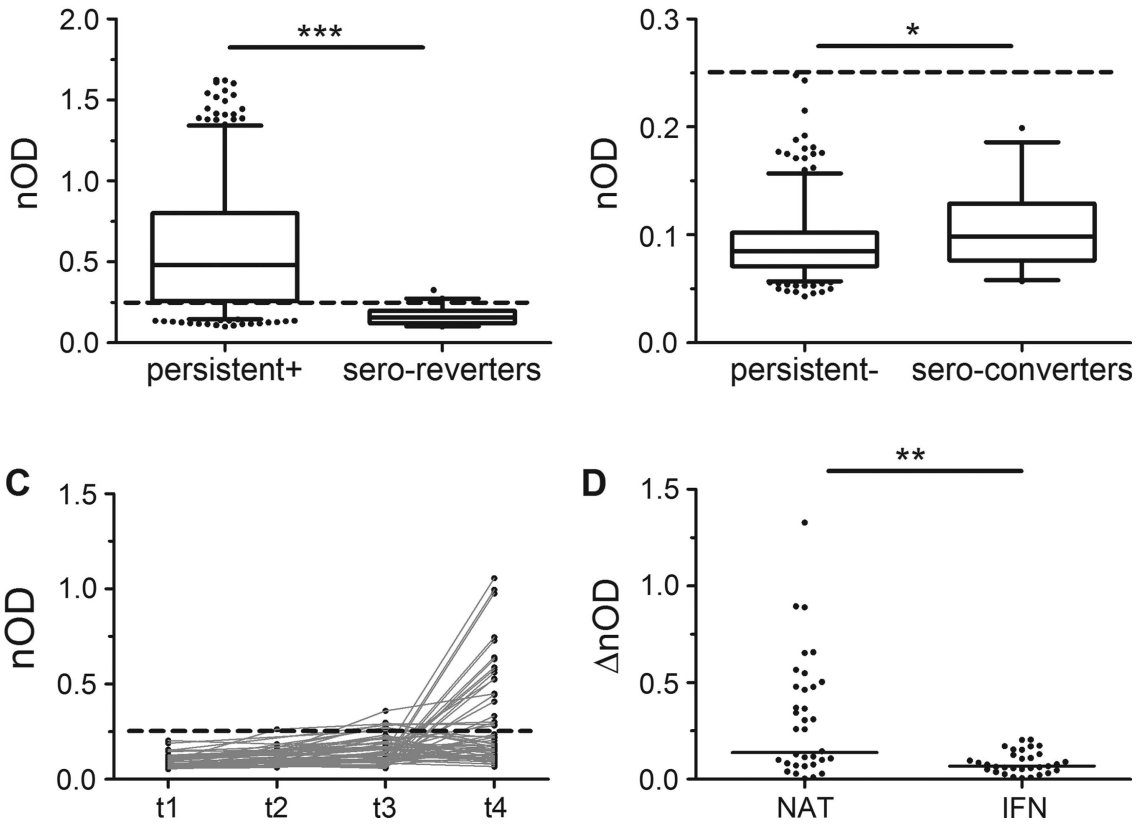

include individuals with primary infection, re-exposure to JCV, or a new site of infection due to the spread of the virus, further studies including subclasses of anti-JCV antibodies are warranted to better understand this observation with regard to its potential relevance to the pathogenesis of PML.

In anti-JCV antibody positive patients the median anti-JCV antibody levels declined slightly after the treatment initiation with NAT (figure 2), while they were unchanged during the preceding therapy with IFN. In addition, a decline in anti-VZV antibody levels was noted after start of treatment with NAT, which was greater compared with the decline observed during IFN therapy (figure 4). Our findings in the IFN-treated patients are in line with published literature, reporting stable anti-JCV antibody levels, ${ }^{21}$ or slightly declining anti-VZV antibodies over time. $^{22}$ By contrast, our data during the NAT therapy is suggestive of a mild suppressive treatment effect on anti-JCV and anti-VZV antibody levels. NAT targets the $\alpha_{4}$-subunit of two important integrins $\left(\alpha_{4} \beta_{1}, \alpha_{4} \beta_{7}\right)$, expressed on $B$ and $T$ cells during various stages of development and maturation in a tissuespecific manner. ${ }^{23}$ These integrins are considered to be
Figure 2 Changes to anti-JCV nOD values in the NAT-group, the IFNsubgroups, and the PML patients. (A) nOD values slightly decreased in anti-JCV positive patients of the NAT-group, were unchanged during IFN therapy (IFN1-subgroup, the IFN2subgroup), or increased in the $5 \mathrm{PML}$ patients. (B) All PML patients increased in $n O D$ by $n O D>0.151$, while only a small proportion of the anti-JCV positive patients showed a similar increase during IFN or NAT therapy.

(C) The median absolute nOD values of the 5 PML patients (black circles and lines) and the NAT-group (grey, depicted as box and whiskers) did not differ at $\mathrm{t} 3$, but were higher at time of PML diagnosis (Dx) compared to the values of the NAT-group at $t 4$. box: interquartile range; horizontal line: median; whiskers: 5-95 percentile. ${ }^{* *} p<0.01 ;{ }^{* * *} p<0.001$ (Kruskal-Wallis test with Dunn's Multiple Comparsion Test or Fisher's exact test).
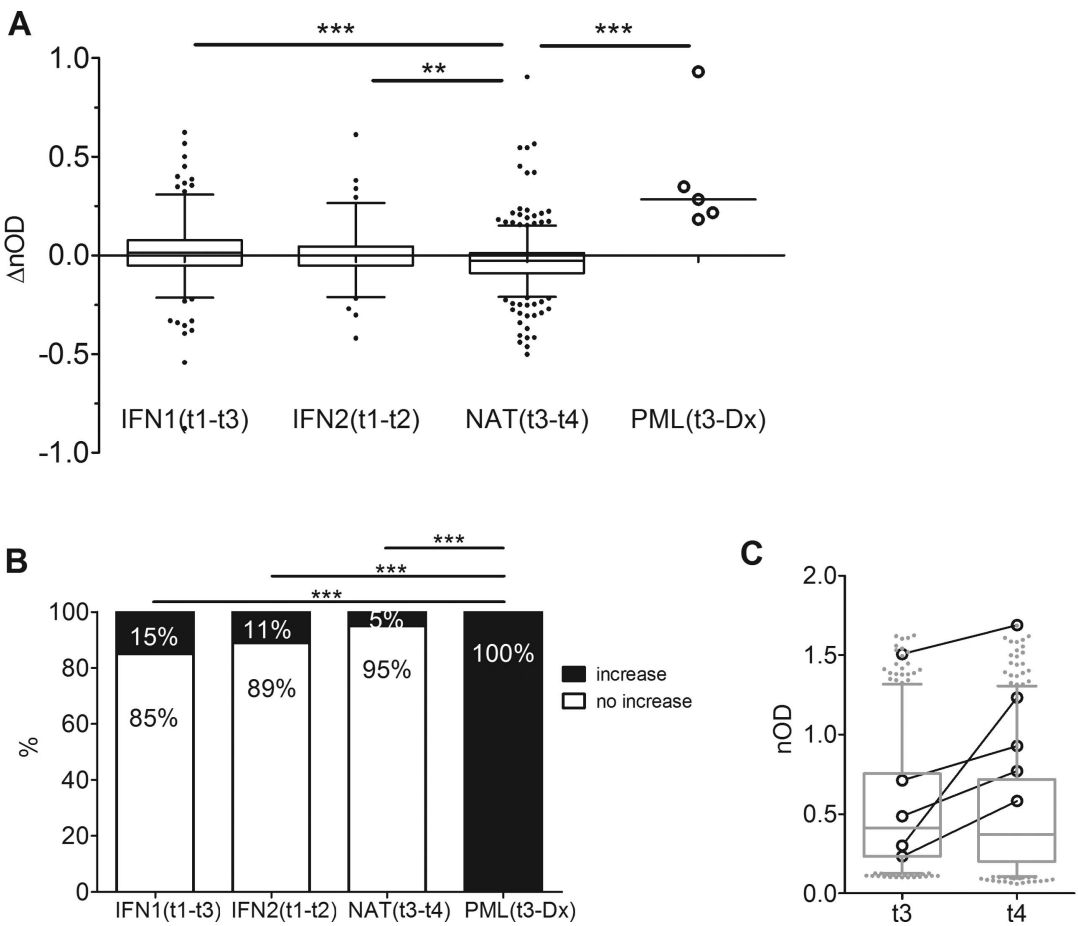
case 1

case 2

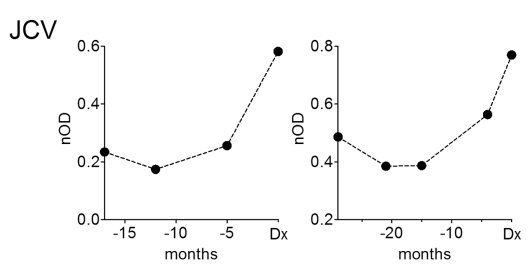

VZV

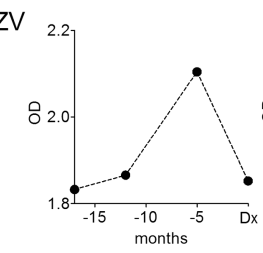

CMV

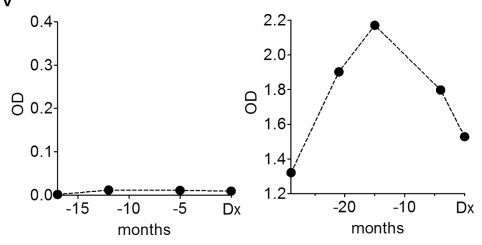

case 3

case 4

case 5
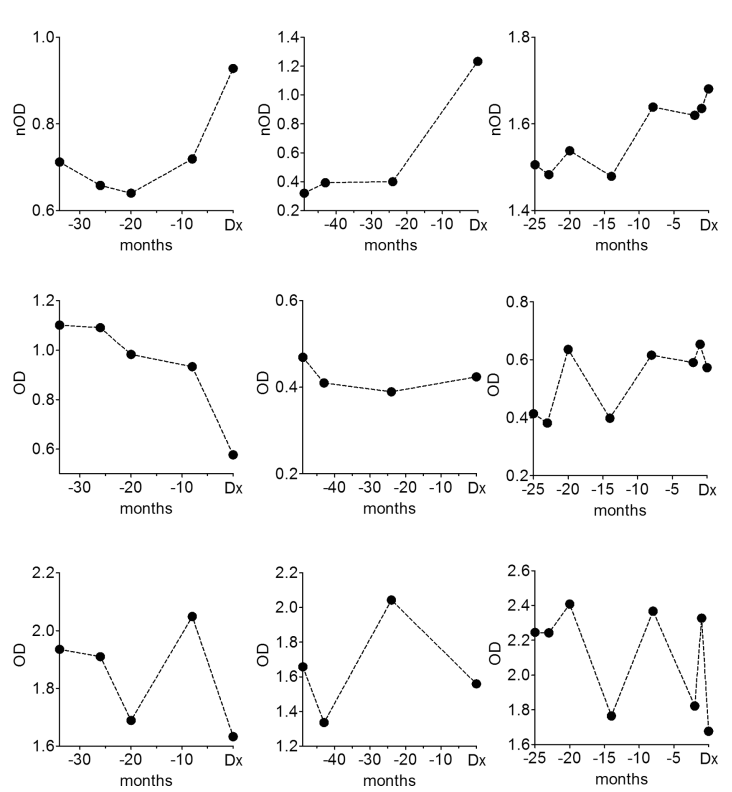

Figure 3 Course of OD values for anti-JCV, anti-VZV, and anti-CMV antibodies in 5 PML patients. For 5 PML cases, optical density (OD) values for anti-JCV, anti-VZV and anti-CMV antibodies are shown, from t3 just before treatment initiation with natalizumab to diagnosis (Dx) of PML, as confirmed by positive JCV PCR in CSF.

important for homing and adhesion of leukocytes to their niches. Various groups have reported changes to B cell homeostasis in the peripheral blood during NAT treatment, with higher numbers of immature pre-B cells and memory and marginal zone like B cells being found in the peripheral blood compartment. ${ }^{24-29}$ Importantly, impaired homing of these cells to their natural niches might be associated with a loss of prosurvival stimuli relevant to the persistence and quality of the antibody response to recall-antigens. ${ }^{30} \mathrm{~A}$ suppressive mechanism affecting the humoral memory response, but not the response toward a neoantigen, could potentially explain our findings: increases in nOD after seroconversion in anti-JCV antibody negatives, as well as a mild decline in antibody levels in the antibody positives. Therefore, further studies assessing the humoral memory function might contribute to further elucidate the pathogenesis of PML during therapy with NAT.

By contrast with the findings for anti-JCV and anti-VZV antibodies, anti-CMV antibodies increased slightly during NAT treatment (figure 4). This could potentially be explained by the different natural kinetics of the antibody responses to different viruses, as previously reported ${ }^{22}$ : compared with JCV and VZV,
CMV causes a blood-borne persistent infection that induces a strong antiviral antibody response, with increasing titres years after infection, ${ }^{31} 32$ and higher median anti-CMV antibody levels in the elderly. ${ }^{33}$ The blood-borne persistent infection with CMV might be associated with a higher frequency of subclinical reactivations, and thus, a different mechanism to maintain the immunological memory response.

For anti-JCV antibody positive patients, the overall downwards trend in antibody levels was associated with a significantly reduced proportion of patients increasing in anti-JCV antibody levels (figure 2). This is of importance, as at time of PML diagnosis, all five Swedish patients with PML showed an upwards trend in anti-JCV antibodies, while anti-VZV and anti-CMV antibodies did not follow these kinetics (figure 3). Therefore, we conclude that the onset of PML can be accompanied by an increase in anti-JCV antibody levels in serum, possibly driven by a new site of infection with a lytic character, and/or the pathogenic transformation of the virus (figure 3). In line with our findings, an increase in anti-JCV antibody levels in serum during the course of PML has been previously observed. ${ }^{34}$ Trampe et $a l^{35}$ recently reported on nine patients with pre-PML samples studied for anti-JCV antibodies: all nine
Figure 4 Changes to anti-VZV and anti-CMV OD values in the NAT-group, the IFN-subgroups, and the PML patients. (A) change in optical density $(\triangle O D)$ values from the anti-VZV ELISA of the NAT-group, the IFN2- subgroup, and the 5 PML patients. (B) change in optical density $(\triangle O D)$ values from the anti-CMV ELISA of the NAT-group, the IFN2-subgroup, and the $5 \mathrm{PML}$ patients. box: interquartile range; horizontal line: median; whiskers: 5-95 percentile. ns: non-significant; $* * * p<0.001$ (Kruskal-Wallis test with Dunn's Multiple Comparsion test).
A

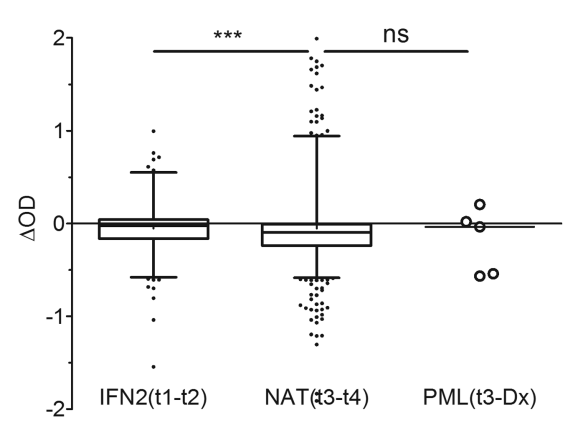

B

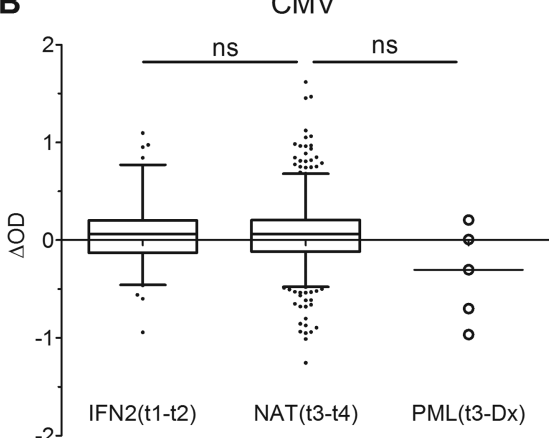


had relatively high levels of anti-JCV antibodies (nOD>0.6) before PML diagnosis, and of the four with more than one pre-PML sample, three showed an increase in nOD values over time. In our cohort, while only $2 / 5$ patients had absolute nOD values $>0.6,100 \%$ of the patients (with $95 \%$ specificity) had mild to moderate increases in nOD compared with a baseline value in the same individual prior to start with therapy (figures 2 and 3). We therefore conclude that the monitoring of anti-JCV antibodies for high levels prior to $\mathrm{NAT}^{35}$ or increasing levels during the therapy with NAT (our data), could potentially allow the prediction or earlier diagnosis of PML, at least in a proportion of the anti-JCV positive patients.

There are limitations to our large-scale national study: our cut-off for an increase in nOD values is empirically set based on the changes to anti-JCV antibodies in a cohort of NAT-treated anti-JCV positive patients with a median time between sampling of only 12 months (range 1-38 months), and only five cases of PML, all diagnosed after the first year of therapy. Considering the fact that the anti-JCV antibody positive patients who did not develop PML were sampled only once during the NAT therapy, and the time between sampling was shorter compared with the PML cases, the natural fluctuations of anti-JCV antibody levels not predictive for the development of PML most likely are not entirely reflected in our study. In addition, although the changes observed were highly consistent, and the virus-specificity of the increase in anti-JCV antibodies for the PML cases was supported by anti-VZV and anti-CMV antibodies not changing, the increases were mild, and just above our empirically defined threshold in two of the five PML cases. Therefore, our data should ideally be confirmed in a prospective longitudinal multicentre trial, including a larger number of PML patients, and controls. Such a study might be well worth performing: in combination with high clinical vigilance and regular follow-up imaging, changes to the anti-JCV antibody levels in serum in the antibody positive risk cohort could broaden our armamentarium in the PML risk surveillance of patients treated with NAT. Since the measurement of anti-JCV antibody levels only requires a serum sample that can be taken at the time of a scheduled infusion, further evaluation of this approach to stratify patients for higher or lower risk of PML could be relatively straightforward.

\footnotetext{
Acknowledgements The analysis for anti-JCV antibodies was sponsored by Biogen Idec. Dr Warnke is supported by an ECTRIMS fellowship stipend, and the KI foundation (2011FoBi1135). Dr Olsson has received grant support for MS research from the Swedish Research Council, EU fp6, Neuropromise and Euratools, Söderbergs Foundation and Bibbi and Niels Jensens foundation. Dr Bergström is supported by VR-M (grant no 521-2011-3297) and LUA-ALF (grant ALF Gbg-145841). Dr Warnke, Dr Fogdell-Hahn and Dr Kieseier received support from the Innovative Medicines Initiative Joint Undertaking under grant agreement $n^{\circ}$ [115303], resources of which are composed of financial contribution from the European Union's Seventh Framework Programme (FP7/2007-2013) and EFPIA companies' in kind contribution. Dr. Kieseier is supported by grants from the German Ministry for Education and Research (BMBF, "German Competence Network Multiple Sclerosis" (KKNMS), Natalizumab-Pharmakovigilanzstudie, 01GI1002). The communicating author highly appreciates the excellent help of Anna Mattsson, Elin Engdahl, Malin Lundkvist, Christina Hermanrud, Helga Westerlind, Alexandra Ahlberg, Roger Jungedal, Jenny Link, Izaura Lima Bomfim, Belghis Davoudi, Veronika Bedarova, Lea Mascarell Maricic, Agneta Carlsson, Maria Johansson, Ingegerd Löfving Arvholm, Katharina Fink, Charlotte Dahle, Anna-Lena Hammarin and Benita Zweygberg-Wirgart.
}

Contributors Study concept and design: CW, TO, SG, JH and AF-H. Acquisition and analysis of data: CW, RR, TB, SG, TP, MS, AR, IK, CH and AF-H. Statistical analysis: CW and RR. Drafting of manuscript: CW and AF-H. Critical revision of the manuscript for important intellectual content: TO, JH and AF-H.

Competing interests Dr Warnke is funded by an ECTRIMS (European Committee for Treatment and Research in Multiple Sclerosis) fellowship stipend and received travel expenses for attending conferences from Teva Pharma GmbH. Dr Kockum,
Dr Bergström, Ms Holmen and Dr Rahbar declare no conflict of interest. Dr Plavina and Dr Subramanyam are employees of Biogen Idec and hold stocks with Biogen Idec. Dr Goelz is an employee of Elan Pharmaceuticals and holds stocks with Elan Pharmaceuticals and Biogen Idec. Dr Kieseier has received personal compensation for activities with Bayer Schering, Biogen Idec, Genzyme, Merck Serono, Novartis, Roche, Sanofi Aventis Pharmaceuticals, Talecris and Teva Neuroscience. Dr Kieseier has research support from Bayer Schering, Biogen Idec, Merck Serono and Teva Neuroscience. Dr Hillert has received unrestricted research support from Biogen Idec, MerckSerono and Bayer Schering. Dr Olsson has received grant support for MS research from the Swedish Research Council, EU fp6, Neuropromise and Euratools, Söderbergs Foundation and Bibbi and Niels Jensens foundation. Unrestricted grant support has been received from Biogen Idec, Bayer, SanofiAventis and Merck. Lecture fees and/or advisory board consultancies for the same companies. Dr Fogdell-Hahn has received unrestricted research support from Biogen Idec, MerckSerono, and Bayer Schering. The analysis for anti-JCV antibodies was performed and sponsored by Biogen Idec.

Ethics approval Karolinska Institutet regional ethical committee (KIDnr 2006/1:7, 2006-08-23).

Provenance and peer review Not commissioned; externally peer reviewed.

Open Access This is an Open Access article distributed in accordance with the Creative Commons Attribution Non Commercial (CC BY-NC 3.0) license, which permits others to distribute, remix, adapt, build upon this work non-commercially, and license their derivative works on different terms, provided the original work is properly cited and the use is non-commercial. See: http://creativecommons.org/ licenses/by-nc/3.0/

\section{REFERENCES}

1 Coisne C, Mao W, Engelhardt B. Cutting edge: natalizumab blocks adhesion but not initial contact of human $T$ cells to the blood-brain barrier in vivo in an animal model of multiple sclerosis. J Immunol 2009;182:5909-13.

2 Rice GP, Hartung HP, Calabresi PA. Anti-alpha4 integrin therapy for multiple sclerosis: mechanisms and rationale. Neurology 2005;64:1336-42.

3 Clifford DB, De Luca A, Simpson DM, et al. Natalizumab-associated progressive multifocal leukoencephalopathy in patients with multiple sclerosis: lessons from 28 cases. Lancet Neurol 2010;9:438-46.

4 Kappos L, Bates D, Edan G, et al. Natalizumab treatment for multiple sclerosis: updated recommendations for patient selection and monitoring. Lancet Neurol 2011;10:745-58

5 Yousry TA, Major EO, Ryschkewitsch C, et al. Evaluation of patients treated with natalizumab for progressive multifocal leukoencephalopathy. $N$ Engl J Med 2006:354:924-33.

6 Sunyaev SR, Lugovskoy A, Simon K, et al. Adaptive mutations in the JC virus protein capsid are associated with progressive multifocal leukoencephalopathy (PML). PLoS Genet 2009; 5:e1000368.

7 Gorelik L, Reid C, Testa M, et al. Progressive multifocal leukoencephalopathy (PML) development is associated with mutations in JC virus capsid protein VP1 that change its receptor specificity. J Infect Dis 2011;204:103-14.

8 Reid CE, Li H, Sur G, et al. Sequencing and analysis of JC virus DNA from natalizumab-treated PML patients. J Infect Dis 2011;204:237-44.

9 Warnke C, Menge T, Hartung HP, et al. Natalizumab and progressive multifocal leukoencephalopathy: what are the causal factors and can it be avoided? Arch Neurol 2010;67:923-30.

10 Gorelik L, Lerner M, Bixler S, et al. Anti-JC virus antibodies: implications for PML risk stratification. Ann Neurol 2010;68:295-303.

11 Goelz SE, Gorelik L, Subramanyam M. Assay design and sample collection can affect anti-John Cunningham virus antibody detection. Ann Neurol 2011;69:429-30; author reply 30-1.

12 Bloomgren G, Richman S, Hotermans $C$, et al. Risk of natalizumab-associated progressive multifocal leukoencephalopathy. N Engl J Med 2012:366:1870-80.

13 Warnke C, Adams 0, Hartung HP, et al. Risk stratification of progressive multifocal leukoencephalopathy under natalizumab: recommendations for JC virus serology. Nervenarzt 2011:82:1314-19.

14 Bozic C, Richman S, Plavina T, et al. Anti-John Cunnigham virus antibody prevalence in multiple sclerosis patients: baseline results of STRATIFY-1. Ann Neurol 2011;70:713-21.

15 Plavina $T$, Berman $M$, Njenga $M$, et al. Multi-site analytical validation of an assay to detect anti-JCV antibodies in human serum and plasma. J Clin Virol 2012;53:65-71.

16 Holmen C, Piehl F, Hillert J, et al. A Swedish national post-marketing surveillance study of natalizumab treatment in multiple sclerosis. Mult Scler 2011;17:708-19.

17 Piehl F, Holmen C, Hillert J, et al. Swedish natalizumab (Tysabri) multiple sclerosis surveillance study. Neurol Sci 2011;31(Suppl 3):289-93.

18 Schmitz H, Doerr HW, Kampa D, et al. Solid-phase enzyme immunoassay for immunoglobulin M antibodies to cytomegalovirus. J Clin Microbiol 1977:5:629-34. 
19 Grahn A, Studahl M, Nilsson S, et al. Varicella-Zoster Virus (VZV) Glycoprotein E Is a Serological Antigen for Detection of Intrathecal Antibodies to VZV in Central Nervous System Infections, without Cross-Reaction to Herpes Simplex Virus 1. Clin Vaccine Immunol 2011;18:1336-42.

20 Thomsson E, Persson L, Grahn A, et al. Recombinant glycoprotein E produced in mammalian cells in large-scale as an antigen for varicella-zoster-virus serology. J Virol Methods 2011;175:53-9.

21 Knowles WA, Pipkin P, Andrews N, et al. Population-based study of antibody to the human polyomaviruses BKV and JCV and the simian polyomavirus SV40. J Med Virol 2003:71:115-23.

22 Amanna IJ, Carlson NE, Slifka MK. Duration of humoral immunity to common viral and vaccine antigens. N Engl J Med 2007:357:1903-15.

23 Leite RP, Carmo-Fonseca M, Cabecadas J, et al. Differential expression of cell adhesion molecules in the functional compartments of lymph nodes and tonsils. Clin Mol Pathol 1995;48:M93-100.

24 Bonig $\mathrm{H}$, Wundes $\mathrm{A}$, Chang $\mathrm{KH}$, et al. Increased numbers of circulating hematopoietic stem/progenitor cells are chronically maintained in patients treated with the CD49d blocking antibody natalizumab. Blood 2008:111:3439-41.

25 Jing D, Oelschlaegel U, Ordemann R, et al. CD49d blockade by natalizumab in patients with multiple sclerosis affects steady-state hematopoiesis and mobilizes progenitors with a distinct phenotype and function. Bone Marrow Transplant 2010:45:1489-96

26 Saure C, Warnke C, Zohren F, et al. Natalizumab and impedance of the homing of CD34+ hematopoietic progenitors. Arch Neurol 2011:68:1428-31.
27 Warnke C, Smolianov V, Dehmel T, et al. CD34+ progenitor cells mobilized by natalizumab are not a relevant reservoir for $\mathrm{JC}$ virus. Mult Scler 2011;17:151-6.

28 Zohren F, Toutzaris D, Klarner V, et al. The monoclonal anti-VLA-4 antibody natalizumab mobilizes CD34+ hematopoietic progenitor cells in humans. Blood 2008; 111:3893-5.

29 Planas R, Jelcic I, Schippling S, et al. Natalizumab Treatment Perturbs Memory- and Marginal Zone-like B-cell Homing in Secondary Lymphoid Organs in Multiple Sclerosis. Eur J Immunol 2011;42:790-8.

30 Koopman G, Keehnen RM, Lindhout E, et al. Adhesion through the LFA-1 (CD11a/CD18)-ICAM-1 (CD54) and the VLA-4 (CD49d)-VCAM-1 (CD106) pathways prevents apoptosis of germinal center B cells. J Immunol 1994;152:3760-7.

31 Amanna IJ, Slifka MK. Mechanisms that determine plasma cell lifespan and the duration of humoral immunity. Immunol Rev 2010;236:125-38.

32 Flo RW, Nilsen A, Voltersvik $\mathrm{P}$, et al. Serum antibodies to viral pathogens and Toxoplasma gondii in HIV-infected individuals. Apmis 1993;101:946-52.

33 Vescovini R, Biasini C, Telera AR, et al. Intense antiextracellular adaptive immune response to human cytomegalovirus in very old subjects with impaired health and cognitive and functional status. J Immunol 2010;184:3242-9.

34 Ryschkewitsch CF, Jensen PN, Monaco MC, et al. JC virus persistence following progressive multifocal leukoencephalopathy in multiple sclerosis patients treated with natalizumab. Ann Neurol 2010;68:384-91.

35 Trampe AK, Hemmelmann C, Stroet A, et al. Anti-JC virus antibodies in a large German natalizumab-treated multiple sclerosis cohort. Neurology 2012;78:1736-42. 